\title{
Références bibliographiques du dossier « Éducation et ruralités "
}

Bernadette Plumelle

\section{OpenEdition}

1 Journals

Édition électronique

URL : http://journals.openedition.org/ries/2294

DOI : 10.4000/ries.2294

ISSN : 2261-4265

Éditeur

Centre international d'études pédagogiques

Édition imprimée

Date de publication : 1 avril 2012

Pagination : 125-135

ISBN : 978-2-85420-594-7

ISSN : 1254-4590

Référence électronique

Bernadette Plumelle, «Références bibliographiques du dossier «Éducation et ruralités » », Revue internationale d'éducation de Sèvres [En ligne], 59 | avril 2012, mis en ligne le 01 avril 2012, consulté le 30 avril 2019. URL : http://journals.openedition.org/ries/2294 ; DOI : 10.4000/ries.2294

(c) Tous droits réservés 


\section{Références \\ bibliographiques}

\section{Bernadette Plumelle}

Le sujet de l'éducation en milieu rural concerne toutes les parties du monde. Les problématiques sont différentes selon qu'il s'agit de pays développés ou de pays en développement mais tous rencontrent des difficultés. Cette bibliographie n'a pas l'ambition de fournir l'exhaustivité des études sur ce sujet. Les références proposées, très récentes en majorité, ont été sélectionnées en vue d'apporter une vue d'ensemble de la littérature sur ce sujet. La bibliographie est organisée en quatre parties. Après quelques données générales sur la ruralité dans le monde, une partie centrale propose des documents sur l'éducation en milieu rural avec des études régionales et nationales. Les parties suivantes proposent une sélection comportant des exemples de programmes d'appui au développement de l'éducation en zone rurale, complétés d'études de cas destinées à illustrer la variété des actions menées en direction de ces publics. Une sélection de sites complète la bibliographie.

Bibliographie arrêtée le 20 février 2010.

\section{LA POPULATION RURALE DANS LE MONDE}

United Nations: Division population, Rural population, development and the environment 2011, Nations unies/New York, 2011, 2 p. http://www.un.org/ [en ligne]

Ce tableau de données sur la population rurale dans le monde présente les données disponibles sous forme de quinze indicateurs et fournit des estimations aux niveaux national, régional et mondial.

\section{GUIBERT Martine, JEAN Yves (sous la direction de), Dynamiques des espaces ruraux dans le monde, Armand Colin/Paris, 2011, 408 p.}

Trois milliards d'habitants sont ruraux, avec une grande diversité selon les régions. Ainsi, au Brésil, $16 \%$ de la population est rurale alors qu'en Inde, elle est de $70 \%$ et de $50 \%$ en Asie du Sud-Est. L'ouvrage se décline en deux parties. Après un article introductif apportant des considérations d'ordre épistémologique sur l'évolution des concepts mobilisés par les géographes, la première partie se focalise sur les défis majeurs auxquels les espaces ruraux sont confrontés particulièrement en rapport avec l'urbain. La seconde partie regroupe des études régionales (Afrique sub-saharienne; Europe du Nord, de l'Ouest, Europe centrale et orientale ; Asie du Sud-Est ; Chine ; Inde ; Brésil).

\section{LUGINBÜHL Yves (sous la direction de), Nouvelles urbanités, nouvelles ruralités en Europe, Éditions P.I.E. Peter Lang/Bruxelles, 2007, 532 p.}

De nouveaux espaces transnationaux, réseaux de villes, interactions villes/campagnes et cohésions régionales apparaissent et de nouvelles inégalités sociales et spatiales se développent. L'ouvrage, qui fait suite à un colloque organisé au Conseil de l'Europe en 2000, propose une quarantaine d'études qui montrent que villes et campagnes ont réagi tout à fait différemment aux effets des bouleversements mondiaux, et que les acteurs sociaux contournent et inventent une infinie variété de relations à l'espace, à l'économie et à la nature. 
OCDE, Le nouveau paradigme rural : Politiques et gouvernance, OCDE/Paris, 2007, $172 p$.

Le rapport récapitule les principales tendances socio-économiques observées dans les zones rurales des pays de l'OCDE; il évoque les besoins qu'engendre une approche transsectorielle de la politique rurale en termes de gouvernance.

\title{
L’ÉDUCATION EN ZONE RURALE
}

\author{
ALPE Yves, FAUGUET Jean-Luc, Sociologie de l'école rurale, L'Harmattan/Paris, \\ 2008, $212 p$.
}

Les débats sur le système éducatif en milieu rural ont marqué l'histoire du discours social sur l'éducation et restent encore présents. La première partie présente les grandes orientations du débat au travers de trois thèmes : citoyenneté, aménagement du territoire et inégalité des chances. Un éclairage sur des situations étrangères complète l'analyse (Afrique, Québec, Catalogne, Roumanie). La deuxième partie propose une synthèse des résultats de la recherche menée par l'Observatoire de l'école rurale en France (1999-2005). La troisième partie élargit le cadre de recherche autour de la question controversée de la «forme scolaire rurale » et de la spécificité du lien social dans l'espace rural.

\section{FRISIRAS Christine M.E (ed.), Rural education in the 21st century: education in a competitive and globalizing world, Nova Science Pub Inc/New York, 2009, $246 p$.}

Les écoles rurales diffèrent considérablement les unes des autres, et elles sont différentes selon les pays. L'ouvrage propose des analyses de la situation des écoles en milieu rural au Danemark, en Grèce, en Argentine, en Afrique du Sud et aux États-Unis (un chapitre est consacré aux programmes de développement des écoles de ce pays).

\section{JEAN Yves (sous la direction de), Géographies de l'école rurale : acteurs, réseaux et territoires, Ophrys/Paris, 2007, 303 p.}

La scolarisation en espace rural constitue à la fois un enjeu éducatif et d'aménagement du territoire. L'école rurale connaît aujourd'hui des formes d'organisation qui varient du maintien de l'école de village aux regroupements concentrés ou à la mise en réseau d'écoles dispersées. Les contributeurs analysent principalement la situation de l'école rurale en France, mais plusieurs chapitres font le point sur ce qui se passe au Québec, en Catalogne et au Portugal. La dernière partie, consacrée aux innovations pédagogiques, aborde les questions de la circulation de l'information, des rapports au savoir et du sens des actes pédagogiques.

LUGINBÜHL Odile, PELLEGRINI Dario (coordinateurs), "L'école en milieu rural : dossier ", Revue internationale d'éducation Sèvres, juin 1996, $n^{\circ}$ 10, p. 23-137, bibliogr.

L'école en milieu rural a fait l'objet, dans de nombreux pays, de débats passionnés car le monde rural change et avec lui le secteur scolaire qui y est implanté. Les contributions de ce numéro s'organisent autour de deux grandes orientations : l'adaptation de ce secteur scolaire face aux transformations du monde contemporain ; la réflexion sur son fonctionnement spécifique et son rôle dans le milieu environnant. L'examen de situations de l'école rurale en France, en Russie, au Portugal, en Norvège, en Italie mettent en lumière des constantes, qu'elles se manifestent dans les préoccupations des enseignants et partenaires de l'école comme dans les solutions proposées par les responsables des systèmes éducatifs. 


\title{
L'ÉDUCATION EN ZONE RURALE : ÉTUDES RÉGIONALES ET NATIONALES
}

\author{
DIAS DA GRACA Patricia, SAUVAGEOT Claude, Indicateurs pour la planification \\ de l'éducation pour les populations rurales : un guide pratique, IIPE/Paris, \\ 2005, 80 p.
}

Le programme de "partenariat sur l'éducation pour les populations rurales » vise à combler le fossé entre les zones urbaines et rurales ainsi qu'à renforcer l'accès des populations rurales à l'éducation de base et à améliorer la qualité de l'éducation. Le guide présente les principes et techniques nécessaires pour élaborer des outils spécifiques de suivi et de pilotage des stratégies d'éducation rurale et propose un ensemble d'indicateurs. Il aborde principalement le niveau national mais il donne également certaines indications pour l'élaboration d'indicateurs similaires au niveau régional.

WATKINS Kevin (sous la direction de), Rapport mondial de suivi sur l'EPT 2010 : atteindre les marginalisés, UNESCO/Paris, 2010, 538 p., statistiques.

Dans ce rapport, l'Unesco définit les facteurs qui contribuent à la marginalisation et à l'absence de possibilités éducatives : le sexe, le revenu et le lieu de résidence se conjuguent à d'autres facteurs comme la langue, l'appartenance ethnique et le handicap. La faible densité de la population oblige la plupart du temps les enfants des zones rurales à effectuer un long trajet pour rejoindre l'école. Le rapport fournit de nombreuses données statistiques concernant des pays d'Afrique (Burkina Faso, Cameroun, Éthiopie, Malawi, Niger, Sénégal et Zambie), d'Asie (Chine, Laos) et d'Amérique du Sud, qui mettent en lumière les effets de l'éloignement géographique sur la scolarisation.

ZHANG Yanhong, POSTLETHWAITE Neville T., GRISAY Aletta (ed.), A view inside primary schools: A world education indicators (WEI) cross-national studies, UNESCO-UIS/Montréal, 2008, 291 p. disponibles sur le site http://www. uis.unesco.org [en ligne]

L'Institut international de statistiques de l'UNESCO présente les résultats d'une enquête menée dans onze pays d'Amérique latine, d'Asie et d'Afrique du Nord. Le questionnaire a porté sur le fonctionnement des établissements scolaires, sur les méthodes pédagogiques, sur les conditions d'apprentissage et sur l'aide dont bénéficient les enseignants et les chefs d'établissements. L'étude met en évidence la forte incidence des inégalités sociales sur les systèmes éducatifs primaires dans de nombreux pays et montre les importants écarts de ressources entre les écoles urbaines et rurales.

\section{Afrique}

ADEA : association pour le développement de l'éducation en Afrique/Paris, FAO : organisation des Nations Unies pour l'alimentation et l'agriculture/Rome, L'éducation pour les populations rurales en Afrique : rapport d'un séminaire ministériel - Addis Abéba Éthiopie 7-9 septembre 2005, IIPE/Paris, ADEA/Paris, FAO/Rome, 2007, 85 p. disponibles sur le site http://unesdoc.unesco.org [en ligne] L'accès des populations rurales, souvent pauvres et analphabètes, à une éducation de qualité est un défi majeur pour atteindre les objectifs d'éducation pour tous (EPT). Pour relever ce défi, un programme a été lancé en septembre 2002, "Éducation pour les populations rurales » et un séminaire régional a été organisé. Le rapport s'appuie sur ces conclusions pour analyser les besoins des populations rurales dans leurs contextes éducatifs très divers et proposer des solutions en matière de planification et de pilotage. 
MINGAT Alain, NDEM Francis, Développer le premier cycle secondaire : enjeu rural et défi pour l'Afrique subsaharienne, AFD: Agence française de développement/Paris, mai 2010, 88 p. disponibles sur le site http://www.cefeb.org [en ligne]

La dimension rurale des scolarisations est explorée sous plusieurs angles. En premier lieu, les auteurs établissent les principales données factuelles concernant la couverture scolaire, puis ils examinent l'offre de services éducatifs en milieu rural et urbain du point de vue des modes d'organisation et des coûts. Enfin, ils abordent la question des impacts économiques de l'éducation.

\section{Amérique du nord}

STRANGE John, JOHNSON Jerry, SHOWALTER Daniel, et al., Why rural matters 2011-12: the conditions of rural education in the 50 states, Rural school and community trust/Washington, janvier 2012, 10 p. disponibles sur le site http:// files.ruraledu.org [en ligne]

En 2008-2009, plus de 9,6 millions d'élèves des écoles publiques aux États-Unis (un quart des enfants américains) ont été inscrits dans des écoles rurales des districts et les inscriptions s'y font à un rythme plus rapide que dans les zones urbaines. Le rapport a pour objectif de fournir des informations et des analyses qui mettent en évidence les besoins prioritaires des écoles publiques rurales et des communautés ainsi que la complexité des situations.

Conseil supérieur de l'éducation/Québec, L'éducation en région éloignée : une responsabilité collective, mars 2009, Ministère de l'éducation du loisir et du sport/ Québec, 137 p. disponibles sur le site http://www.cse.gouv.qc.ca [en ligne]

Dans les régions éloignées du Québec, la baisse démographique conjuguée à la dispersion des communautés sur de grands territoires et à la fragilité de leur situation socioéconomique pose des défis éducatifs majeurs. Le Conseil supérieur de l'éducation trace un portrait de six régions considérées comme "éloignées » et fait état des actions entreprises par les acteurs locaux et régionaux. Il propose quatre orientations : accessibilité à des services éducatifs de qualité ; harmonisation de l'offre de formation avec les besoins des personnes et des communautés; renforcement du leadership des régions; adaptation des interventions de l'État aux besoins des régions.

\section{Amérique latine}

FAO, UNESCO, CIDE : centro de investigación y desarrollo de la educación de Chile, Educación para la población rural en Brasil, Chile, Colombia, Honduras, México, Paraguay y Peru, FAO/Rome, 2004, 443 p. disponibles sur le site: http:// unesdoc.unesco.org [en ligne]

Le rapport présente des études sur la situation et les politiques éducatives menées dans sept pays d'Amérique latine. La population rurale est de 14 à $30 \%$ (50\% pour le Honduras et le Paraguay). Chaque étude comprend un diagnostic de la situation, les tendances et des recommandations. La couverture éducative s'est améliorée au niveau du secteur primaire mais reste très faible dans le secondaire. Selon les auteurs, le manque d'homogénéité de la population rurale est l'un des principaux facteurs qui expliquent le retard de l'éducation, les habitants des zones côtières, montagneuses ou dans la jungle ayant des besoins différents qui devraient être pris en compte par les programmes scolaires. 
QUEVEDO CAMACHO Rafael Isidro, "La educación y la capacitación rural en la región andina », Agroalimentaria, $n^{\circ} 21$, juillet-décembre 2005, p. 93-112 disponibles sur le site http://www.redetis.org.ar [en ligne]

L'auteur analyse la situation des programmes éducatifs et de formation des zones rurales dans les Andes (Amérique du sud), pour identifier les principaux changements dans les politiques nationales et les institutions internationales en matière de développement éducatif.

\section{Asie}

\section{Chine}

TENG FU Margaret, "Les inégalités d'accès à l'enseignement primaire entre urbains et ruraux ", Perspectives chinoises, $n^{\circ}$ 89, mai-juin 2005 disponibles sur le site http://perspectiveschinoises.revues.org [en ligne]

Cet article traite des inégalités dans l'accès à l'enseignement primaire entre urbains et ruraux. Il rappelle ce qu'est le système éducatif "à deux voies ", et montre comment ce dernier a abouti à un déséquilibre en termes d'accès à l'éducation.

\section{THOGERSEN Stig, A County of Culture: Twentieth Century China Seen from} the Village Schools of Zouping, Shandong, The University of Michigan Press, 2002, 320 p.

L'auteur présente les stratégies mises en œuvre dans les campagnes chinoises pour transformer la société rurale par l'éducation, à travers l'étude d'un district ordinaire du nord de la Chine, Zouping. Il dresse un panorama retraçant les efforts menés tout au long du vingtième siècle pour transformer la vie rurale par des réformes de l'éducation. Il met en lumière les divergences d'opinion sur ces politiques entre les réformateurs de l'éducation, les agents de l'État, les enseignants et les parents, ainsi que la mise en place d'initiatives.

ZHAO Dan, PAROLIN Bruno, "School Mapping Restructure in China: What Role for the Small Rural School? ", Frontiers of Education in China, vol. $6 n^{\circ} 2$, p. 248-278, juin 2011

Malgré des progrès, ces quinze dernières années, dus à la restructuration de la carte scolaire (School Mapping Restructure: SMR), de nombreuses petites écoles rurales ont été fermées ou ont fusionné avec de plus grandes écoles primaires. L'auteur examine le rôle des petites écoles rurales dans le système éducatif chinois, regarde le contexte et les impacts de SMR sur ces écoles en termes d'indicateurs de qualité, de coût et d'accès.

\section{Inde}

BHATTACHARJEA Suman, WADHWA Wilima, BANERJI Rukmini, Inside primary schools: a study of teaching and learning in rural India, Assessment survey evaluation research (ASER)/India, octobre 2011, 108 p. disponibles sur le site : http://images2.asercentre.org [en ligne]

Une étude sur l'enseignement et l'apprentissage dans l'Inde rurale a permis de suivre environ 30000 enfants dans différents États indiens pendant quinze mois. Elle a porté sur l'organisation scolaire, la formation des enseignants et les modalités d'enseignement en classe ainsi que les résultats d'apprentissage. Les équipes de recherche se sont également rendues dans les maisons des enfants, pour comprendre en quoi les caractéristiques sociales, économiques et éducatives des familles affectent leur apprentissage. 
OLIVEAU Sébastien, CHASLES Virginie, "Sur les chemins de l'école : accessibilité et alphabétisation dans le monde rural sud-indien », 2005, Espace populations sociétés, p. 453-464 disponibles sur le site http://eps.revues.org [en ligne]

Les auteurs ont étudié l'alphabétisation à partir d'une base de données reprenant des données du recensement. Après avoir remis en contexte l'alphabétisation au niveau de l'Inde, ils proposent une exploration à l'échelle villageoise de quatre États du sud du pays, en rappelant les facteurs socioéconomiques (développement, religion, etc.) et en insistant sur trois déterminants des inégalités : le niveau de centralité des villages, l'accessibilité des structures éducatives et enfin leur qualité.

\section{Europe}

FERREIRA Jorge, "Primary school challenges in the rural areas of Finland, France and Portugal ", Education et sociétés, 2011, $n^{\circ} 28$, p. 109-130

Comment la France, la Finlande et le Portugal définissent-ils et gèrent-ils le maillage des écoles primaires en milieu rural? Quel degré d'autonomie chaque pays accorde-t-il aux autorités locales pour organiser l'école? L'auteur a analysé les instructions officielles dont la comparaison révèle l'opposition entre la très forte décentralisation finlandaise et un système centralisé caractérisé au Portugal. La France apparaît dans une position médiane. Il montre que les choix politiques de l'État influent sur la typologie des modes d'organisation des établissements. Alors qu'en Finlande et en France plusieurs modèles coexistent, un seul s'impose au Portugal.

JENTSCH, Birgit et Mark SHUCKSMITH (dir.), Young people in rural areas of Europe, Ashgate/Aldershot, 2004, 334 p.

L'ouvrage présente une étude comparative portant sur l'expérience des jeunes en milieu rural en Autriche, en Finlande, en France, en Irlande, en Allemagne, au Portugal et au Royaume-Uni, qui s'appuie sur les résultats d'une recherche subventionnée par la Commission européenne (projet PAYPIRD). Cette étude souligne le rôle des écoles, du développement communautaire et de la culture locale, ainsi que la tension entre une société de l'individualisation et la famille, les amis, l'église et les réseaux au sein de la communauté, dans la formation de trajectoires d'insertion professionnelle des jeunes ruraux.

\section{France}

ALPE Yves, "Existe-t-il un "déficit culturel" chez les élèves ruraux ? ", Revue française de pédagogie, septembre 2006, $n^{\circ} 156$, p. 75-88

L'institution scolaire considère que les difficultés rencontrées par les élèves issus de l'école et du collège rural ont pour origine un isolement géographie et social provoquant un "déficit culturel ", qui expliquerait la moindre réussite scolaire de ces élèves. Les recherches menées dans le cadre de l'Observatoire de l'école rurale depuis 1999 remettent en cause ce diagnostic ; elles montrent aussi que l'école, et peut-être plus encore le collège, ne jouent pas le rôle culturel qui leur est assigné dans la recherche de l'égalité des chances.

ALPE Yves, FAUGUET Jean-Luc, " Enseigner dans le rural : un "métier" à part ?", Travail et formation en éducation, 2008, $n^{\circ} 2$, disponibles sur : http://tfe.revues. org/index767.html [en ligne]

Le métier d'enseignant en zone rurale s'exerce dans des conditions différentes de celles qui prévalent dans la plupart des écoles urbaines. Peut-on parler pour autant d'un "métier » de l'enseignant en zone rurale? Les performances des élèves sont davantage marquées par l'origine socioprofessionnelle des parents que par des effets de territoires. Par ailleurs, la spécificité des pratiques pédagogiques relève plus d'une différence de degré que d'une hétérogénéité de nature, et les écarts observés sont aussi importants entre enseignants ruraux qu'entre les maîtres du rural et de l'urbain. 
CHAMPOLLION Pierre, LEGARDEZ Alain, "Mieux prendre en compte les contextes territoriaux de l'école pour mieux adapter la formation des enseignants aux évolutions de la société et des élèves : l'exemple des espaces et territoires ruraux et montagnards ", in BAILLAT Gilles, NICLOT Daniel, ULMA Dominique (sous la direction de), La formation des enseignants en Europe: approche comparative, De Boeck Université/Bruxelles, 2010, p. 267-287

Les auteurs prônent la prise en compte des contextes spatiaux et territoriaux dans la formation professionnelle des enseignants afin de mieux adapter celle-ci aux évolutions de la société et des élèves.

DUHAMEL Marcel, HOUCHOT Alain, CUBY Jean-François, MOULIN Yves, L'évolution du réseau des écoles primaires, Ministère de la Jeunesse, de l'Éducation nationale et de la recherche/Paris, 2003, 71 p.

En France, les écoles primaires sont incitées depuis des années à se fédérer dans des réseaux multiformes. Cette évolution concerne aussi bien les écoles des communes rurales que celles des métropoles urbaines. Dans ce rapport, les inspections générales (IGEN, IGAENR) ont effectué des enquêtes dans des territoires dont certains sont en zone rurale (la Côte d'Or, le Calvados, le Tarn), afin de faire le point sur l'organisation et la structure des écoles.

GRELET Yvette, VIVENT Céline, "La course d'orientation des jeunes ruraux ", Bref du CEREQ, septembre 2011, $n^{\circ}$ 292, 4 p. disponibles sur le site http://www. cereq.fr [en ligne]

Les parcours scolaires des jeunes ruraux se distinguent de ceux des urbains par des études moins longues, une préférence marquée pour l'enseignement professionnel, des ambitions scolaires plus réduites. Ces disparités s'expliquent par l'environnement socio-économique et la faiblesse de l'offre de formation en zone rurale.

VIEILLARD-BARON Hervé, LAFOND Viviane de, MATHIEU Nicole, et al. « Le rural : terre d'exclusion?", Ville-école-intégration, septembre 2003, $n^{\circ} 134,253$ p.

La campagne en France est globalement dans une dynamique démographique positive mais il existe des zones de désertification dans certains départements et l'accroissement d'une forme de marginalisation de certains territoires. Ce numéro propose tout d'abord un état des lieux des campagnes, avec les phénomènes d'exclusion que peut connaître la jeunesse. La deuxième partie a pour sujet l'école et son rôle dans l'aménagement du territoire. La dernière partie concerne des politiques rurales comme le projet d'écoles rurales en réseaux ou une étude des Maisons familiales rurales.

\section{LES PROGRAMMES D'APPUI AU DÉVELOPPEMENT DE L’ÉDUCATION EN MILIEU RURAL}

ACKER David, GASPERINI Lavinia, Education for rural people: the role of education training and capacity development in poverty reduction and food security, FAO/Rome, mai 2009, 156 p. disponibles sur le site: http://www.fao.org/ [en ligne]

Ce livre est le résultat de l'examen de collaborations entre la FAO, l'Unesco et environ trois cent cinquante partenaires liés au Cadre d'action de Dakar sur l'Éducation pour tous et du World Food Summit Plan of Action. Il a été élaboré pour aider les décideurs politiques à relever les défis que représente l'éducation des populations rurales. Il couvre l'éducation formelle et non formelle, l'alphabétisation ainsi que la formation des compétences et propose un certain nombre d'exemples dans différentes parties du monde. 
FAO / Unité de coordination du partenariat éducation pour la population rurale (EPR), Les ONG françaises et le programme FAO/UNESCO (EPR), FAO : Organisation des Nations unies pour l'alimentation et l'agriculture/Rome, 2005, 76 p. disponibles sur le site: http://www.fao.org [en ligne]

Le programme «Éducation pour les populations rurales » vise à donner accès à l'éducation de base aux populations rurales, en renforçant le partenariat entre tous les acteurs de l'éducation. Il s'inscrit dans une double logique de lutte contre la pauvreté et de sécurité alimentaire. Sous forme de fiches-projets, ce document constitue un panorama commenté des initiatives de vingt-sept ONG françaises en matière d'éducation formelle et non formelle.

IWG : International Working Group on Education, Advancing in education: reaching rural people, developing capacity: international working group on Education (IWGE) Rome Italy, 12-14 june 2006, UNESCO-IIPE/Paris, 2007, 173 p. disponibles sur le site http://unesdoc.unesco.org [en ligne]

Ce livre est une synthèse des questions examinées durant la réunion du Groupe international de travail sur l'éducation (GITE) tenu à la FAO en 2006. Il s'agit d'un outil de formation pour les initiatives de renforcement de développement conçu pour les planificateurs et les éducateurs sur le développement rural. Il met l'accent sur les disparités dans la fourniture de l'éducation et calcule les coûts pour assurer la réalisation de l'objectif de l'éducation primaire universelle. Il analyse également comment améliorer l'accès à l'éducation dans les régions rurales.

\title{
Programmes internationaux et nationaux
}

\begin{abstract}
Alliance for excellent education, Current challenges and opportunities in preparing rural high schools students for success in college and careers: what federal policymakers need to know, Alliance for excellent education/Washington, février 2010, 48 p. disponibles sur le site http://www.all4ed.org [en ligne]

Plus d'un cinquième de deux mille écoles secondaires les moins performantes des ÉtatsUnis sont situées dans les régions rurales; les taux de décrochage scolaire sont élevés et les taux d'inscription trop faibles. Actuellement, un élève sur quatre en milieu rural ne parvient pas à terminer ses études secondaires. Ce rapport d'un think tank américain offre une analyse des défis auxquels sont confrontées les écoles secondaires rurales : les financements, les standards de qualité, l'aide financière aux élèves ou le recrutement d'enseignants qualifiés.
\end{abstract}

CEFRIO: centre francophone d'informatisation des organisations/Québec, L'école éloignée en réseau (EER) un modèle : rapport synthèse septembre 2011, novembre 2011, 37 p. disponibles sur le site http://www.cefrio.qc.ca [en ligne]

Ce rapport de recherche présente huit années d'expérimentation (2002-2010) du programme " école éloignée en réseau (ÉÉR) ", conçu comme alternative à la fermeture des petites écoles rurales au Québec ne disposant pas des ressources locales garantissant la qualité de l'environnement d'apprentissage. Il s'agit d'un dispositif de télécollaboration par Internet pour les petites écoles rurales. Les chapitres offrent une vue d'ensemble des effets de ce dispositif sur l'apprentissage des élèves, la qualité des échanges entre pairs, les connaissances et habiletés des enseignantes et des enseignants, l'organisation de la classe, les conditions d'innovation présentes dans l'école et dans la commission scolaire. 
CHINAPAH Vinayagum, Education for rural transformation (ERT): national international and comparative perspectives, Stockholm university: Institute of international education/Stockholm, novembre 2011, 311 p. disponibles sur le site http://www.interped.su.se [en ligne]

Le rapport fait suite au colloque international, tenu en Suède en novembre 2010, dont les contenus ont porté sur les concepts, les pratiques et les politiques éducatives mises en œuvre pour la transformation du monde rural. Dix-huit communications ont été rassemblées. La première partie offre une perspective internationale sur les concepts et les outils développés avec plusieurs études concernant la Tanzanie, le Népal et l'Europe centrale et orientale. Les parties suivantes concernent la Chine et l'Inde, pays dans lesquels les écarts entre les mondes rural et urbain s'accroissent. On y trouve des analyses, des expériences et des études de cas, parmi lesquelles des initiatives d'apprentissage à distance.

LUGAZ Candy, DE GRAUWE Anton, Renforcer le partenariat école-communauté. Résultats d'une recherche au Bénin, au Niger et au Sénégal, IIEPUNESCO/Paris, 2010, 169 p. disponibles sur le site de l'UNESCO http://unesdoc. unesco.org [en ligne]

L'importance des relations école-communauté et la diversité de celles-ci ont amené l'IIPE, des instituts de recherche nationaux et l'ONG Plan à entreprendre une recherche plus détaillée sur les facteurs expliquant et déterminant l'existence d'une bonne relation entre l'école et la communauté. La publication présente les résultats de la recherche menée auprès de structures de représentation des parents, de la communauté et du personnel de l'école au Bénin, au Niger et au Sénégal. La première partie analyse et synthétise les principales leçons de cette recherche. La seconde présente les trois études de cas.

MARTIN Jean-Yves, "Les écoles spontanées en Afrique subsaharienne : champéducatif et contre-champ scolaire ", Cahiers d'études africaines, 2003, $n^{\circ} 169-170, p .19-37$

Des écoles spontanées sont apparues dans différents pays d'Afrique subsaharienne (en particulier Tchad, Mali, Cameroun) au cours des vingt dernières années. Cet article présente une analyse de ce phénomène et en propose une interprétation. Ces écoles spontanées sont replacées dans un cadre historique, de la colonisation aux éducations nationales. Sont ensuite analysées les identités variées de ces écoles selon leurs contextes d'apparition, la nature de la demande qui les a fait naître, le cadre des connaissances transmises, le panorama scolaire où elles s'insèrent et enfin leur statut juridique et l'évolution recherchée.

MULKEEN Aidan, CHEN Dandan (ed.), Teachers for rural schools: experiences in Lesotho, Malawi, Mozambique, Tanzania and Uganda, World Bank publications/ Washington, 2008, 138 p.

Les écoles, dans les endroits difficiles à atteindre, peinent à attirer et à retenir des enseignants. Aussi la gestion et l'appui aux enseignants dans ces régions nécessitent-elles une attention particulière. L'ouvrage examine toutes ces questions et propose des études de cas au Lesotho, au Malawi, au Mozambique, en Tanzanie et en Ouganda, qui donnent un aperçu du potentiel et des inconvénients des différentes options choisies.

MULKEEN Aidan G., HIGGINS Cathal, Multigrade teaching in Sub-Saharan Africa : lessons from Uganda Senegal and the Gambia, World Bank Institute/ Washington, 2009, 42 p. disponibles sur le site http://siteresources.worldbank.org [en ligne]

L'enseignement multigrade est une option importante pour les pays africains qui cherchent à assurer la scolarisation des enfants "hors de l'école" dans les zones à faible densité de population. Dans l'enseignement multigrade, un enseignant travaille avec des élèves de deux 
ou plusieurs niveaux en même temps, dans une seule classe. Pour les écoles observées en Ouganda, les classes réunissaient deux niveaux et ont bénéficié d'un projet pilote (formation des enseignants, matériels d'apprentissage). Au Sénégal, il y avait différents modèles de classes multiniveaux. En Gambie, l'enseignement multigrade a été utilisé en grande partie pour pallier la pénurie d'enseignants.

READING Chris, "Using ICT to increase professional connectedness for teachers in remote Australia ", Australian Educational Computing, vol. 25, $n^{\circ} 2$, décembre 2010, 4 p. disponibles sur le site: http://acce.edu.au [en ligne]

Les enseignants des régions éloignées d'Australie vivent un isolement professionnel. Les collaborations durables avec d'autres enseignants sont difficiles, alors que ce lien est important. Dans un projet national, des TIC ont été utilisées dans cinq États d'Australie pour mettre en contact ces enseignants et pour que se développe un sentiment de communauté.

WARD Michael, "Rural education", in India infrastructure report, Oxford University Press/New Delhi, 2007, p. 286-317 disponibles sur le site : http://dise.in/ [en ligne]

Depuis 2001, le projet Sarva Shiksha Abhiyan («Éducation pour tous») a pour objectif de réintégrer dans l'enseignement primaire vingt millions d'enfants non scolarisés. Les populations ciblées sont les filles, les enfants issus de communautés minoritaires et les enfants handicapés. Ce programme a permis d'importants progrès pour améliorer l'accès à l'éducation préscolaire et primaire dans les zones rurales ces dix dernières années. Le développement des routes a permis ainsi de diminuer l'absentéisme des enseignants dans les écoles de village. Une attention croissante est portée à la gouvernance des écoles, à la formation et au fonctionnement des "village education committes». Le prochain défi consiste à accroître l'accès dans les zones rurales à l'enseignement secondaire, en particulier pour les filles, les «scheduled castes» et les «scheduled tribes».

\section{Études de cas}

BAMBERG Ingrid, "L'école comme centre de vie communautaire : diversification des représentations et des pratiques dans une communauté semi-rurale d'Afrique du sud », Cahiers d'études africaines, 2003, n 169-170, p. 121-142

Pour l'Afrique du sud, le système éducatif décentralisé permet aux acteurs locaux de l'éducation de participer aux prises de décision à travers des comités de gestion élus et composés d'enseignants et les parents. Les stratégies éducatives des parents d'une communauté semi-rurale montrent comment les décisions de ces comités de gestion en matière de frais de scolarité et de langue d'enseignement modèlent l'offre scolaire et influencent le cadre d'interaction possible entre l'école et la communauté.

BLUM Nicole, "Small NGO Schools in India: implications for access and innovation ", Compare: a journal of comparative and international education, vol. 39, $n^{\circ} 2$, mars 2009, p. 235-248

Les ONG jouent un rôle important en Inde dans l'enseignement primaire auprès des communautés défavorisées. Ce document explore les contributions d'un programme qui a cherché à accroître l'accès des enfants marginalisés à l'éducation en fournissant un appui aux petites écoles rurales. Pour l'auteur, les programmes de ce type ont des impacts positifs en termes d'accès et de qualité de l'éducation parce qu'ils sont menés à petite échelle, enracinés localement et les ONG ont une plus grande capacité d'innovation. 


\section{COMPAORE Maxime, Évaluation du programme éducation en milieu pastoral dans le Sahel du Burkina Faso. Evaluation report, Oxfam GB/Oxford, 2008, 39 p.}

Cette étude vise à évaluer le plan d'action de trois ans d'une ONG locale au Burkina Faso, l'association "Nodde Nooto " (A2N). Le programme comportait cinq composantes : accès et rétention à l'école, qualité de l'éducation, promotion de l'équité/genre, renforcement des capacités de la société civile, et appui aux acteurs locaux.

\section{CARE France, Qualité et équité de l'éducation biculturelle du département de} Puno. Pérou, CARE France/Paris, 2011, 14 p.

Ce document présente un projet de l'ONG CARE au Pérou, financé à $75 \%$ par la Commission européenne. Ce projet cherche à repenser l'éducation dans le cadre de l'éducation interculturelle bilingue. Il s'agit d'améliorer la qualité de l'apprentissage des élèves inscrits dans les établissements interculturels bilingues et de renforcer les capacités de gestion pédagogique des fonctionnaires régionaux et locaux, éducateurs et instituteurs.

CASELY-HAYFORD Leslie, HARTWELL Ash, "Reaching the underserved with complementary education: lessons from Ghana's state and non-state sectors ", Development in Practice, vol. 2, $n^{\circ} 4-5$, juin 2010, p. 527-539

Entre 1995 et 2006, plus de 85000 enfants âgés de 8 à 14 ans ont participé à un programme d'éducation complémentaire dans des zones rurales du nord du Ghana. School for Life, une ONG, a proposé neuf mois d'instruction dans la langue parlée par les enfants. Une évaluation de l'impact du programme a démontré les effets positifs : atteinte d'un niveau d'alphabétisme de base dans leur langue maternelle plus rapidement et de manière plus économique que dans les écoles primaires de l'État.

LAMARKBI Nadia, LAFAYE DE MICHEAUX Elsa, "L'ouverture de l'éducation primaire rurale aux ONG. Les enseignements du Maroc contemporain », Mondes en développement, $n^{\circ} 134,2006, p$. 79-94

Dans un contexte d'analphabétisme et de sous-scolarisation des enfants de paysans, le gouvernement marocain a fait appel en 1998 aux ONG pour assurer la formation des élèves de zones rurales isolées. À partir d'une enquête de terrain, cet article étudie l'émergence d'un nouveau marché scolaire et tente de cerner les contours de l'enseignement donné aux élèves.

LEWANDOWSKI Sophie, "Les savoirs locaux au Burkina Faso : enjeux pédagogiques et sociaux ", Ethnologie française, décembre 2007, $n^{\circ} 4$, p. 605-612

Dans l'éducation rurale au Burkina Faso, l'usage des contes est l'occasion pour les jeunes de s'interroger sur le réel visible et invisible. Mais tel n'est pas le projet de l'école classique, qui nie l'existence des savoirs locaux. Sont apparues de nouvelles écoles utilisant les langues locales et développant une culture censée allier savoirs scolaires et savoirs locaux. Pourtant ces savoirs métissés restent dépendants de choix et de conditions matérielles d'enseignement difficiles à généraliser.

PRIGENT Steven, " La faible scolarisation en milieu rural cambodgien s'expliquet-elle seulement par la pauvreté ?", Cahier de la recherche sur l'éducation et les savoirs, $n^{\circ} 10,2011$, p. 63-83

$\mathrm{Au}$ Cambodge, l'offre scolaire est peu structurée alors qu'elle est, selon l'auteur, fondamentale pour réussir une politique de scolarisation volontariste. Si les enfants ne vont pas à l'école, ce n'est pas pour des raisons d'éloignement, ni parce que les maîtres ne sont pas respectés ou à cause de frais de scolarité. Selon l'auteur, les seuls motifs de la faible scolarisation sont à rechercher du côté de la demande. Au Cambodge, la décision scolaire relève bien plus d'un niveau local que national. La scolarisation y est une affaire de famille. 
